\title{
On the non-thermal high energy radiation of galaxy clusters
}

\author{
A. N. Timokhin ${ }^{1}$, F. A. Aharonian ${ }^{2}$, and A. Yu. Neronov ${ }^{3}$ \\ 1 Sternberg Astronomical Institute, Universitetskij pr. 13, Moscow, 119992, Russia \\ 2 Max-Planck-Institut für Kernphysik, Saupfercheckweg 1, Heidelberg, 69117, Germany \\ 3 Institut de Physique Théorique, Université de Lausanne, BSP 1015 Lausanne, Switzerland
}

Received 2 May 2003 / Accepted 26 November 2003

\begin{abstract}
The origin of the non-thermal EUV and hard X-ray emission "excess" reported from some galaxy clusters has been intensively debated over the last several years. The most favored models which refer this excess to relativistic electrons upscattering the $2.7 \mathrm{~K}$ Cosmic Microwave Background Radiation (CMBR) generally requires a very low magnetic field, significantly below the estimates derived from the Faraday Rotation Measurements, unless one invokes rather nonstandard assumptions concerning the energy and pitch angle distributions of non-thermal electrons. In this paper we suggest a new model assuming that the "non-thermal" excess is due to synchrotron radiation of ultra-relativistic (multi-TeV) electrons of "photonic" origin. These electrons are continuously introduced throughout the entire intracluster medium by very high energy (hypothetical) $\gamma$-rays through interactions with the diffuse extragalactic radiation fields. We present numerical calculations for the Coma cluster, and briefly discuss implications of the model for other galaxy clusters both in the $\mathrm{X}$ - and $\gamma$-ray energy domains.
\end{abstract}

Key words. X-rays: galaxies: clusters - galaxies: clusters: individual: Coma - gamma rays: theory

\section{Introduction}

Galaxy clusters - the largest gravitationally bound structures in the Universe - contain many hundreds or thousands member galaxies surrounded by diffuse hot gas with temperatures close to $\sim 10^{8} \mathrm{~K}$. Correspondingly, these objects are characterized by intensive thermal X-ray emission. At the same time, non-thermal processes connected to acceleration and radiation of relativistic particles play a non-negligible role in the energy budget of these objects. In particular, diffuse radio emission with a steep energy spectrum and low surface brightness is observed from many galaxy clusters (Giovannini et al. 1999). Polarization measurements provide strong evidence for the synchrotron origin of this emission. The recent reports of the extreme-ultraviolet (EUV) "excess" emission and hard X-radiation (HXR) from some galaxy clusters represent new, although less firmly established, evidence for non-thermal activity in galaxy clusters.

The first systematic search for non-thermal X-ray emission from six galaxy clusters by HEAO-1 resulted only in upper limits (Rephaeli \& Gruber 1988). However, the recent studies with RXTE and BeppoSAX satellites revealed a possible non-thermal X-ray component from a few clusters of galaxies. While there is clear evidence for non-thermal X-rays from the Coma cluster (Fusco-Femiano et al. 1999; Rephaeli \& Gruber 2002), the claims of the presence of non-thermal X-rays in the spectra of other objects, e.g. Abell 2199 (Kaastra et al. 1999)

Send offprint requests to: F. A. Aharonian, e-mail: Felix. Aharonian@mpi-hd.mpg.de and Abell 2256 (Fusco-Femiano et al. 2000), need further confirmation. The detection of diffuse "excess" radiation at lower energies (in the EUV band) from Coma (Lieu et al. 1996; Bowyer et al. 1999) and Virgo (Bowyer et al. 1996; Berghöfer et al. 2000) was initially interpreted as thermal radiation of the warm intracluster medium, but soon it became clear that this radiation has most likely a non-thermal origin (see e.g. Berghöfer \& Bowyer 2002). Later, using the data of BeppoSAX LECs observations, Kaastra et al. (1999) reported detection of nonthermal emission from Abell 2199, however Bowyer et al. (1999); Berghöfer \& Bowyer (2002) argued that this, as well as the claims of "excess" EUV emission from some other galaxy clusters (except for Coma and Virgo) was not adequately justified. On the other hand, the community seems quite confident about the detection of non-thermal high energy radiation from the Coma cluster in both EUV and X-ray bands.

The radio and EUV/X-ray emission components from galaxy clusters could be tightly coupled, i.e. produced by the same population of electrons through the synchrotron and inverse Compton (IC) channels of radiation, respectively (Rephaeli 1977). Since the main target photons for the inverse Compton scattering are provided by the $2.7 \mathrm{~K} \mathrm{CMBR}$, the ratio of the radio and EUV/HXR fluxes depends significantly $\left(\propto B^{-2}\right)$ on the intracluster magnetic field (ICMF), but is rather insensitive to the specific energy distribution of electrons. For the last several years the inverse Compton origin of the EUV/HXR "excess" has been explored by many authors (e.g. Hwang 1997; Bowyer \& Berghöfer 1998; Sarazin \& Lieu 1998; Atoyan \& Völk 2000; Petrosian 2001; Brunetti et al. 2001; 
Tsay et al. 2002). The general conclusion of these studies is that if one interprets EUV and HXR emission as a result of inverse Compton scattering of electrons responsible for diffuse radio emission, the ICMF cannot significantly exceed $0.1-0.2 \mu \mathrm{G}$, unless one introduces quite unusual assumptions concerning the energy spectrum and the pitch angle distributions of electrons. This is an order of magnitude lower than follows from Faraday Rotation Measures (RM) for several galaxy clusters (Clarke et al. 2001). Although this discrepancy could be somewhat reduced within more sophisticated IC models and by adequate treatment of observational selection effects (Petrosian 2001; Rephaeli \& Gruber 2002), it is important to search for alternative approaches and mechanisms to explain the EUV/HXR excess. Atoyan \& Völk (2000) and Tsay et al. (2002) have shown that formally it is possible to accommodate, within the two-component IC models, the EUV excess from Coma even with a high intracluster magnetic field. At the same time, they demonstrated that the hard X-ray emission cannot be reproduced by any inverse Compton scenario if the magnetic field exceeds a few $\mu \mathrm{G}$. It is clear that for explanation of hard X-ray emission from Coma one needs alternative radiation mechanism(s).

In particular, it has been suggested that HXR might be explained by bremsstrahlung of a supra-thermal electron population (Enßlin et al. 1999; Blasi 2000; Dogiel 2000; Sarazin \& Kempner 2000). This model, however, leads to unreasonably high energy requirements, given the fact that only a negligible $\left(\sim 10^{-6}\right)$ fraction of sub-relativistic electrons is released in the form of bremsstrahlung X-rays (Petrosian 2001). Recently Liang et al. (2002) suggested a revised version of this model assuming quasi-thermal distribution of electrons responsible for X-rays, and argued that this model could partly avoid the abovementioned energetic problem.

In this paper we propose a new scenario for the production of non-thermal EUV and X-ray emission in clusters of galaxies by synchrotron radiation of ultra-relativistic electrons. The key assumption of the model is that these electrons have "photonic" origin, i.e. are produced at interactions of very high energy (VHE) $\gamma$-rays with diffuse extragalactic background radiation. In this way the relativistic electrons are continuously injected throughout the entire intracluster medium, and therefore the resulting synchrotron EUV and X-ray emission should have a diffuse morphology even in the case of a single point source of VHE $\gamma$-rays located in the cluster.

Below we discuss the implications of this hypothesis for high energy non-thermal radiation from clusters of galaxies with some specific calculations for the Coma cluster which shows the strongest evidence for non-thermal emission at EUV and X-ray bands.

\section{The model: Motivation and basic assumptions}

The proposed scenario is based on two assumptions: (i) the non-thermal high energy radiation of galaxy clusters is dominated by synchrotron radiation of ultra-relativistic electrons; (ii) these electrons have non-acceleration origin, namely, they are produced throughout the entire cluster volume in interactions of hypothetical primary very high energy $\gamma$-rays with the diffuse extragalactic radiation fields.
The synchrotron radiation of relativistic electrons is so far the most effective mechanism for the production of $\mathrm{X}$-rays in conditions typical of the intracluster medium (ICM). Indeed, the characteristic time of radiation of synchrotron X-ray photons with energy $E_{\mathrm{X}}: t_{\mathrm{syn}} \simeq 5 \times$ $10^{4}\left(E_{\mathrm{X}} / 1 \mathrm{keV}\right)^{-1 / 2}(B / 1 \mu \mathrm{G})^{-3 / 2} \mathrm{yr}$ is much shorter than any other relevant timescale characterizing radiative and other losses of electrons in these objects. Therefore for production of the EUV/HXR flux $F_{\mathrm{EUV} / \mathrm{X}}$ reported from the Coma cluster at the level of a few times $10^{-11} \mathrm{erg} / \mathrm{cm}^{2} \mathrm{~s}$ a reasonable injection power of multi- $\mathrm{TeV}$ electrons in the ICM is required, $\dot{W}_{\mathrm{e}} \simeq L_{\mathrm{EUV} / \mathrm{X}}=4 \pi d^{2} F_{\mathrm{EUV} / \mathrm{X}} \sim 3 \times 10^{43} \mathrm{erg} / \mathrm{s}$, where $d$ is the distance to the cluster (for Coma $d \sim 100 \mathrm{Mpc}$ ). Note that this estimation is almost independent of the exact value of ICMF as long as the $B$-field exceeds $3 \mu \mathrm{G}$. The current overall energy in these electrons, $W_{\mathrm{e}} \simeq \dot{W}_{\mathrm{e}} t_{\mathrm{syn}}$, does depend on the magnetic field. For $B=3 \mu \mathrm{G}, W_{\mathrm{e}} \sim 10^{55} \mathrm{erg}$. Assuming a broadband (e.g. power-law) injection spectrum of electrons, this estimate can be increased by one or two orders of magnitude (depending on the spectral index and the low energy cutoff in the electron spectrum), but it remains well below the total energy for electrons with energy between $100 \mathrm{MeV}$ and a few $\mathrm{GeV}-W_{\mathrm{e}} \sim \dot{W}_{\mathrm{e}} t_{\mathrm{IC}} \sim 10^{62} \mathrm{erg}$, required by the IC models.

On the other hand, the high efficiency of synchrotron radiation of multi-TeV electrons in the ICMF leads to other problems. It is over-efficient in the sense that the short radiative cooling time of electrons requires an adequate acceleration rate in order to boost electrons to energies well beyond $100 \mathrm{TeV}$. To produce synchrotron photons of energy $E_{\mathrm{X}}$ one needs electrons with energy $E_{\mathrm{e}} \simeq 140\left(E_{\mathrm{X}} / 1 \mathrm{keV}\right)^{1 / 2}(B / 3 \mu \mathrm{G})^{-1 / 2} \mathrm{TeV}$. Although very high energy electrons can be effectively accelerated by strong accretion or merger shocks (Loeb \& Waxman 2000; Inoue \& Sasaki 2001; Miniati 2002; Blasi 2002; Gabici $\&$ Blasi 2003), even for the most favorable conditions (allowing the acceleration to proceed in the Bohm diffusion regime), the maximum electron energy cannot achieve $\geq 100 \mathrm{TeV}$ which is required to explain the non-thermal X-ray spectrum of the Coma cluster reported up to $80 \mathrm{keV}$. Also, the short lifetime does not allow electrons to propagate away from the acceleration sites more than $1 \mathrm{kpc}$. Thus, the diffusive character of the observed EUV and HXR cannot be explained unless we assume continuous (in space and time) production of electrons throughout the cluster. While the direct acceleration of multi-TeV electrons on $\sim 1 \mathrm{Mpc}$ scales in the intracluster medium seems a rather unrealistic scenario, such energetic electrons can be implemented continuously in the cluster as secondary products of interaction of high energy protons and $\gamma$-rays with ambient matter and photon fields (Aharonian 2002).

Since protons in galaxy clusters can be accelerated and effectively confined up to energies $\geq 10^{3} \mathrm{TeV}$ (Volk et al. 1996; Berezinsky et al. 1997) they unavoidably produce relativistic electrons through generation and decay of secondary $\pi^{ \pm}$-mesons. However, because of the low density of the intracluster gas, $n \leq 10^{-3} \mathrm{~cm}^{-3}$, the p-p interaction time exceeds $10^{19} \mathrm{~s}$, therefore the total energy in multi-TeV protons should be at least $10^{63}$ erg in order to explain the EUV and HXR fluxes observed from the Coma cluster by electrons of "hadronic" origin. 


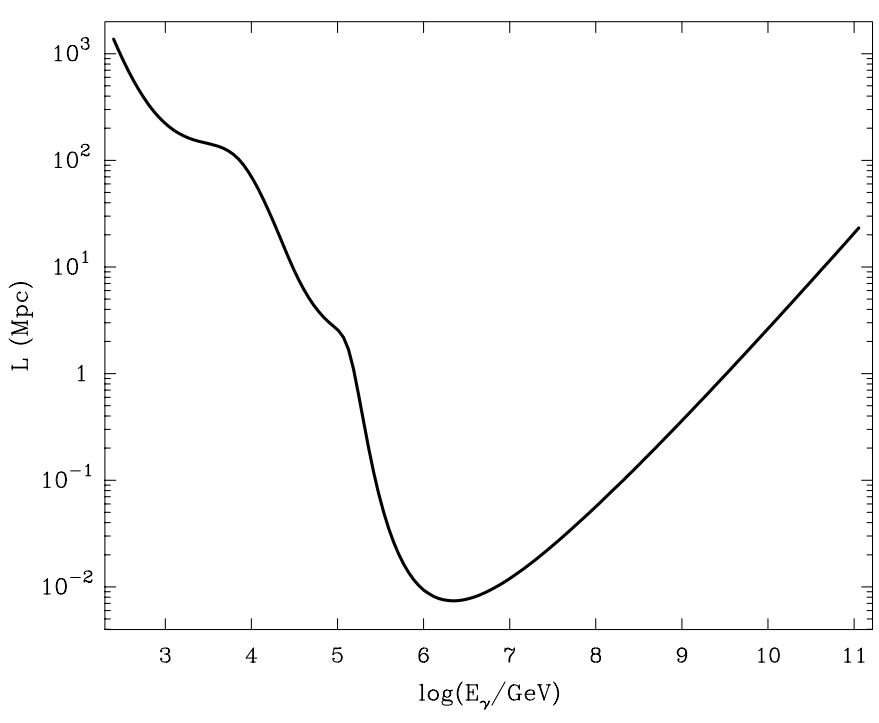

Fig. 1. Mean free path of $\gamma$-rays calculated for the diffuse extragalactic background model described in Aharonian et al. (2002).

The principal assumption of our model is that the ultrarelativistic electrons responsible for the non-thermal high energy emission of a galaxy cluster are products of the interaction of very high energy $\gamma$-rays $\left(10^{14}-10^{19} \mathrm{eV}\right)$ with soft photons of the cosmic diffuse background radiation. The mean free path of such photons is shown in Fig. 1. It varies in the range from 0.1 to $1 \mathrm{Mpc}$, which allows continuous injection of electrons in the entire volume of the cluster. This overcomes two problems that we face in the case of directly accelerated electrons - both related to the short synchrotron cooling time of electrons. The hypothesis of a secondary origin of electrons does not have any intrinsic upper limit on the energy of electrons, and allows implementation of electrons in every point of ICM.

\section{Results}

The energy of the secondary, pair-produced electrons, and therefore the typical energy of their synchrotron radiation, strongly $\left(\propto E^{2}\right)$ depends on the high energy end of the primary gamma-radiation. Below we discuss 3 different cases when spectra of primary $\gamma$-rays extend to (i) "conventional" energies of about $10^{15} \mathrm{eV}$ or less; (ii) to ultra-high energies exceeding $10^{16} \mathrm{eV}$; and (iii) extremely high energies extending to beyond $10^{20} \mathrm{eV}$. Although in all three cases the proposed mechanism works with almost 100 per cent efficiency transforming the energy of primary $\gamma$-rays to synchrotron radiation of pair-produced electrons, the resulting synchrotron radiation appears in different energy bands. We apply this model for the interpretation of the EUV and X-ray "excess" emission from the Coma cluster. Also, we discuss this mechanism in the context of future searches for $\gamma$-rays from clusters of galaxies.

\subsection{Primary $\gamma$-rays with energy less than $10^{15} \mathrm{eV}$}

We assume that a source or an ensemble of sources in the central part of the cluster radiates $\gamma$-rays with a constant rate and energy spectrum given by a "power-law with quasi-exponential cutoff":

$\dot{N}_{\gamma}(E) \propto E^{-\Gamma} \exp \left[\left(E / E_{0}\right)^{-\beta}\right]$.

We use in calculations the energy-dependent mean free path of gamma-rays shown in Fig. 1. We calculate numerically the distribution of electrons and positrons (injection spectrum) produced by $\gamma$-rays from the central source(s) in each point of the cluster. The time-dependent spectra of pair-produced electrons are obtained taking into account their synchrotron and IC energy losses. Finally, spectra of synchrotron and IC radiation of these electrons are calculated. We assume that the secondary, pair-produced electrons are immediately isotropized, we also ignore the propagation effects of these electrons, i.e, assume that the electrons "die", due to severe synchrotron losses, not far from their birthplace. For any reasonable intracluster magnetic field this is an acceptable approximation. The propagation effect may become relatively important for low-energy electrons responsible for radio emission. However, within the assumed model we do not attempt to explain the radio emission, but rather assume that radio emission is due to relatively low energy electrons associated with non-thermal phenomena that took place a long time ago (see e.g. Enßlin \& Sunyaev 2002). At the same time, radio emission produced by the cooled low energy $(\mathrm{MeV} / \mathrm{GeV})$ electrons in the framework of our model should not exceed the observed fluxes. This is an important condition which sets a robust upper limit on the active time of operation of $\gamma$-ray sources and on their energy spectra.

In Figs. 2 and 3 we show the synchrotron and IC spectra of pair-produced electrons in the ICM, calculated for two values of the average ICMF: (a) $B=6 \mu \mathrm{G}$ and (b) $B=0.3 \mu \mathrm{G}$, respectively. For both cases the following parameters of the $\gamma$-ray spectrum are assumed: $\Gamma=2, \beta=1 / 2, E_{0}=700 \mathrm{TeV}$. It is assumed that injection of $\gamma$-rays with a quasi-constant rate into ICM started $10^{7} \mathrm{yr}$ ago. The fluxes shown are corrected for the intergalactic absorption due to interactions with the diffuse extragalactic background radiation fields, adopting the distance to the source of $100 \mathrm{Mpc}$.

Figures 2 and 3 demonstrate that for the chosen combination of parameters it is possible to explain the EUV and X-ray radiation by electrons of "photonic" origin. VHE $\gamma$-ray luminosities of about $2 \times 10^{45}$ and $3 \times 10^{46} \mathrm{erg} / \mathrm{s}$ are required to support the reported EUV and X-ray fluxes for the cases (a) and (b) respectively. Assuming a low energy cutoff in the $\gamma$-ray spectra below $100 \mathrm{TeV}$ one can significantly reduce these energy requirements. In the case (a) the value of the ICMF is in agreement with the estimates derived from RM probes of the Coma cluster (Kim et al. 1990; Feretti et al. 1995). From the point of view of energy requirements, the preference obviously should be given to the case of a strong magnetic field. The case of low magnetic field, which predicts energy flux of the inverse Compton TeV $\gamma$-rays on the level of $\sim(2-3) \times 10^{-10} \mathrm{erg} / \mathrm{cm}^{2} \mathrm{~s}$ (see Fig. 3), is excluded by TeV observation. Such a flux could hardly be missed from the long-term observations of Coma by the HEGRA system of imaging atmospheric Cherenkov telescopes, even taking into account the extended character of this emission. The case of a strong magnetic field predicts significantly reduced secondary $\gamma$-ray flux (Fig. 2), which however 


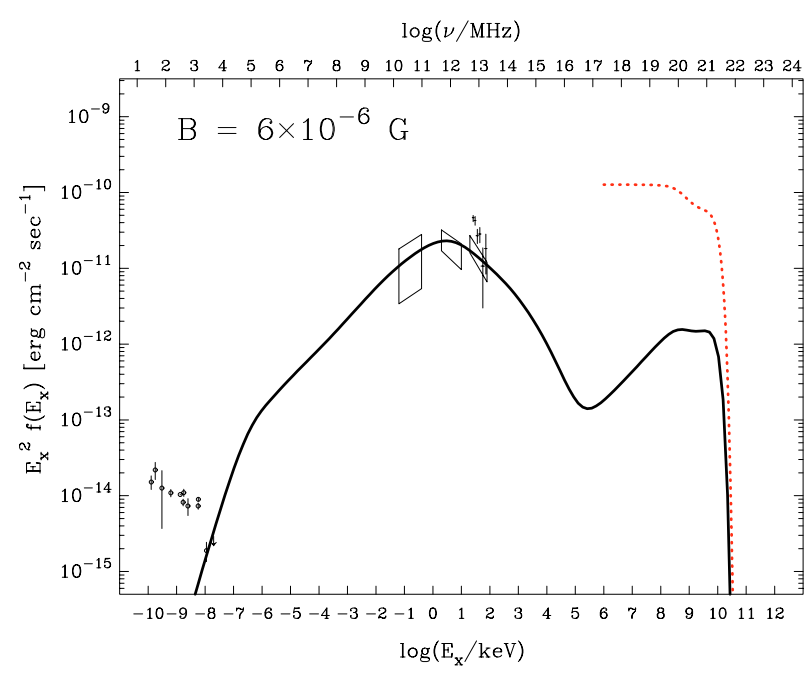

Fig. 2. Spectrum of non-thermal radiation from the Coma cluster (solid line). The radiation is produced via conversion of VHE $\gamma$-rays into electron-positron pairs and subsequent synchrotron and IC radiation of secondary electrons. It is assumed that $\gamma$-rays with spectrum given by Eq. (1) with $\Gamma=2, \beta=0.5, E_{0}=700 \mathrm{TeV}$, have been injected into ICM over the last $10^{7}$ years. The dotted line represents the spectrum of primary $\gamma$-rays as seen by the observer, assuming that $\gamma$-rays are emitted isotropically. Both spectra are corrected for absorption in the intergalactic medium. The compilation of radio data are taken from Deiss et al. (1997). The open boxes in the EUV and soft X-ray domains correspond to the fluxes observed by Lieu et al. (1999). The open box and points in the hard X-ray domain correspond to fluxes reported by Fusco-Femiano et al. (1999). The magnetic field in the cluster is assumed to be $B=6 \mu \mathrm{G}$.

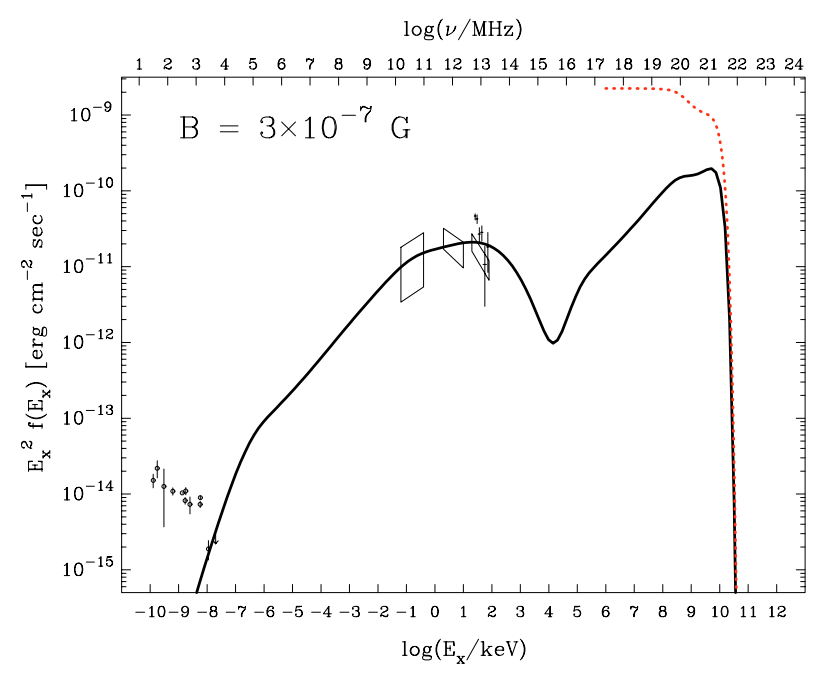

Fig. 3. The same as in Fig. 2, but for the intracluster magnetic field $B=0.3 \mu \mathrm{G}$.

remains sufficiently high, so it can be probed by the next generation of Cherenkov telescope arrays.

In addition to the secondary $\gamma$-rays of IC origin, we should expect also primary $\gamma$-rays, even after intergalactic absorption which becomes significant already at energies above several $\mathrm{TeV}$. The flux of primary $\gamma$-rays, determined by the normalization to provide the EUV and HXR fluxes by synchrotron radiation of secondary electrons, is shown in Figs. 2 and 3

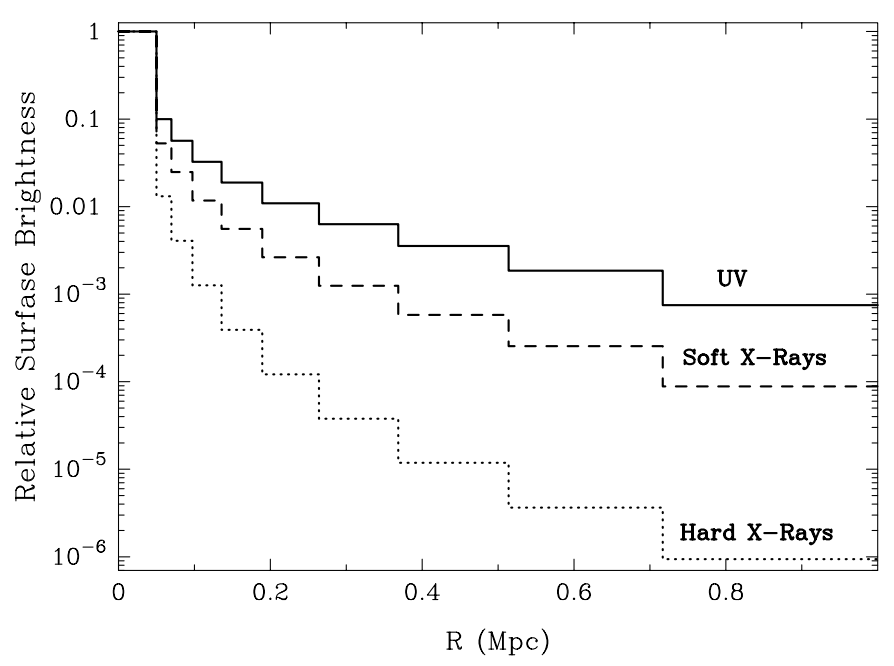

Fig. 4. Relative surface brightness distribution of the non-thermal radiation from Coma cluster in different spectral bands for initial $\gamma$-rays produced in central source. The cluster is divided in 10 zones. Surface brightness is normalized to the brightness of the central zone. In each zone a separate spectrum calculation has been performed. This figure corresponds to the case with large ICMF $(B=6 \mu \mathrm{G})$. Relative surface brightness distribution in EUV energy band (0.064-0.240 keV) is shown by solid line, in Soft X-Rays $(2-10 \mathrm{keV})$ by dashed, and in Hard X-Rays (25-80 keV) by dotted lines.

by a dotted line. It marginally agrees with the EGRET upper limit at $\mathrm{MeV} / \mathrm{GeV}$ energies, and exceeds the sensitivity of the HEGRA telescope system even assuming that the source is extended with an angular size of about 1 degree, or $\gamma$-ray flux is contributed by many sources distributed over the cluster. This discrepancy can be removed if we assume anisotropic $\gamma$-ray emission, e.g. that $\gamma$-rays are contributed by a limited number of AGN with jets away from the direction to the observer. The conflict with the TeV upper limits can be overcome also by assuming that the primary $\gamma$-ray spectrum is harder or contains a low energy cutoff around $100 \mathrm{TeV}$. Such a cutoff could be a result of $\gamma$-ray absorption in the UV/optical/NIR fields inside the primary source(s). While with such an assumption we may dramatically reduce the flux of primary $\gamma$-rays in the observable $(E \leq 10 \mathrm{TeV})$ energy domain, it cannot have a significant impact on the of synchrotron EUV and X-rays, because they are the result of an interaction of $E \geq 100 \mathrm{TeV}$ photons with the diffuse background radiation.

Meanwhile, spatial profiles of the synchrotron radiation of the photoproduced electrons significantly depend both on the ICMF distribution and on the high energy cutoff in the spectrum of primary $\gamma$-rays. The dependence on the flux of the diffuse background radiation is less significant.

In Fig. 4 we show the surface brightness distribution of synchrotron radiation in different energy bands, assuming that the source(s) of primary $\gamma$-rays are concentrated in the central part of the cluster. The profiles are calculated for the same parameters as in Fig. 2. It is seen that with a decrease in photon energy, the brightness distribution becomes broader. This reflects the reduction of the free path of $\gamma$-rays with energy. In this calculation we assume the average intracluster magnetic field of $6 \mu \mathrm{G}$. In reality, the intracluster magnetic field should, of course, 
decrease at larger distances from the center. This should lead to sharper profiles, especially at high energies. On the other hand, if sources of primary $\gamma$-rays are more or less homogeneously distributed in the cluster, we should expect quite flat brightness distributions at all photon energies.

Comprehensive spectral and morphological studies of high energy synchrotron components in the spectra of galaxy clusters can provide a decisive test of the proposed model, and in case of confirmation would reveal unique information about the energy spectrum and angular distribution of "invisible" (absorbed between the source and the observer) $\geq 100 \mathrm{TeV}$ primary $\gamma$-rays.

\subsection{Primary $\gamma$-rays with energy exceeding $10^{16} \mathrm{eV}$}

The energy of a $\gamma$-ray photon interacting with background radiation fields is shared between the secondary electron and positron. However, the major fraction of the energy of the $\gamma$-ray photon is transfered to one of the electrons. Therefore the maximum of the synchrotron radiation of secondary electrons is expected at energy $h v \sim 10(B / 1 \mu \mathrm{G})\left(\mathrm{E}_{\gamma} / 10^{15} \mathrm{eV}\right)^{2} \mathrm{keV}$. Thus, if the spectrum of $\gamma$-rays extends beyond $10^{17} \mathrm{eV}$, the maximum of synchrotron radiation will be shifted to the $\gamma$-ray domain. Therefore, the spectrum of synchrotron radiation dramatically depends on the position of the cutoff energy $E_{0}$ in the primary $\gamma$-ray spectrum. For example, for $E_{0}=10^{16} \mathrm{eV}$, we obtain $h v \sim 1 \mathrm{MeV}$, while for $E_{0}=10^{18} \mathrm{eV}$, the synchrotron maximum appears in the energy range around $10 \mathrm{GeV}$.

This effect is demonstrated in Fig. 5 assuming that primary $\gamma$-rays have an energy distribution given by Eq. (1) with $\Gamma=0 ; \beta=1$, and (i) $E_{0}=10^{16} \mathrm{eV}$, (ii) $10^{18} \mathrm{eV}$ and (iii) $10^{19} \mathrm{eV}$, respectively. Note that this type of $\gamma$-ray spectrum can be formed by ultrahigh energy cosmic ray protons interacting with a narrow-band radiation field. In this case $E_{0}$ is an order of magnitude less than the energy cutoff in the parent proton spectrum. On the other hand, the low energy part of the $\gamma$-ray spectrum $(\mathrm{d} N / \mathrm{d} E=$ const $)$ does not depend on the proton spectrum, but simply is a result of the threshold of photomeson interactions.

The $10^{16}-10^{18}$ eV $\gamma$-ray cannot only be effectively produced in AGN, but also can escape the production regions without catastrophic losses (see e.g. Neronov et al. 2002; Atoyan \& Dermer 2003). Another site for the production of extremely high energy gamma-rays could be the intracluster medium, where the highest energy cosmic rays, $E \geq 10^{20} \mathrm{eV}$, interact with the 2.7 K CMBR (Aharonian 2002). These interactions lead to copious production of secondary $\gamma$-rays, electrons and neutrinos with characteristic energy larger than $10^{19} \mathrm{eV}$.

In Figs. 5a we show the luminosities of synchrotron radiation of secondary electrons. It is seen that if in the case (i) the radiation of the secondary electrons peaks at $\mathrm{MeV}$ energies, in the case (ii) and (iii) the luminosity is dominated by $\mathrm{GeV}$ and $\mathrm{TeV} \gamma$-rays, respectively. However, the very high energy $\gamma$-rays above $1 \mathrm{TeV}$ suffer significant intergalactic absorption if sources are located beyond $100 \mathrm{Mpc}$. To demonstrate this effect, in Figs. 5b we show the expected $\gamma$-ray fluxes after correction for the intergalactic absorption, assuming that the source is located at a distance of $100 \mathrm{Mpc}$. Thus, for the assumed total luminosity in primary $\gamma$-rays of $10^{44} \mathrm{erg} / \mathrm{s}$ and distance to the source of $100 \mathrm{Mpc}$, the resulting $\mathrm{GeV}$ and $\mathrm{TeV} \gamma$-ray fluxes can be probed with GLAST and forthcoming arrays of atmospheric Cherenkov telescopes. At the same time, because of limited sensitivity of $\gamma$-ray instruments in the $\mathrm{MeV}$ energy band, detection of the secondary synchrotron radiation initiated by $10^{16} \mathrm{eV}$ primary $\gamma$-rays would be very difficult, unless the power of primary $\gamma$-rays significantly exceeds $10^{45} \mathrm{erg} / \mathrm{s}$.

\subsection{Non-thermal radiation resulting from decay of super-heavy primordial particles}

In this section we discuss production of secondary electrons by $\gamma$-rays which are not associated with conventional accelerated processes, but are products of decay or interaction of primordial massive particles. These processes constitute the basis of the so-called "top-down" scenarios of production of highest energy cosmic rays (for a review see e.g. Bhattacharjee \& Sigl 2000).

Decay or annihilation of weakly interacting massive particles (WIMP) of mass $m_{\mathrm{X}}$ results in 2 jets of hadrons, with energy $\simeq m_{\mathrm{X}} / 2$ each. The products of hadronic jets are mostly pions $-\pi^{0}, \pi^{+}, \pi^{-}(\sim 32 \%$ in each $)$ with a small fraction of energy released as nucleons $(\sim 4 \%)$. Decays of $\pi$-mesons result in $\gamma$-rays, electrons and neutrinos with mean energy of about $10^{20} \mathrm{eV}$. While neutrinos freely propagate through the intergalactic medium, $\gamma$-rays cannot penetrate deeper than $10 \mathrm{Mpc}$ because of interactions with diffuse extragalactic radio emission. While most of these $\gamma$-rays typically terminate outside of the cluster, the electrons from decays of charged $\pi$-mesons immediately radiate their energy through synchrotron channel in the form of $\gamma$-rays with mean energy $E \simeq 300(B / 3 \mu \mathrm{G})\left(E_{\mathrm{e}} / 10^{20} \mathrm{eV}\right)^{2} \mathrm{TeV}$. These $\gamma$-rays interacting with the $2.7 \mathrm{~K} \mathrm{CMBR}$ and diffuse infrared background photons lead to a new generation of electrons which subsequently produce synchrotron EUV and X-rays as described in the previous section. It is remarkable that both the expected/predicted masses of X-particles and the magnetic fields in the galaxy clusters are in just the "right" regions to yield, though the 2-step electron-photon conversions, a significant fraction of the mass of X-particles in the form of synchrotron EUV and X-radiation. Detailed calculations of this radiation require good knowledge of spectral distributions of secondary particles or the so-called fragmentation functions. The simplest form corresponding to a very flat spectrum of particles is approximated as $\mathrm{d} N_{\mathrm{i}} / \mathrm{d} x \propto$ $x^{3 / 2}$, where $x=2 E_{\mathrm{i}} / m_{\mathrm{X}}$ is the dimensionless energy of the decay product of type i. Besides this "crude" approximation (called below as fragmentation function 1) in the present study we used four other, theoretically better-developed approximations proposed by Hill (1983) (model 2), Berezinsky et al. (1997) (model 3), Berezinsky \& Kacherliess (2001) (model 4) and by Birkel \& Sarkar (1998) (model 5).

We performed calculations for several combinations of the intracluster magnetic field strength $B$ and the mass of $\mathrm{X}$-particle $m_{\mathrm{X}}$ to find the best fit to the reported fluxes. Results 

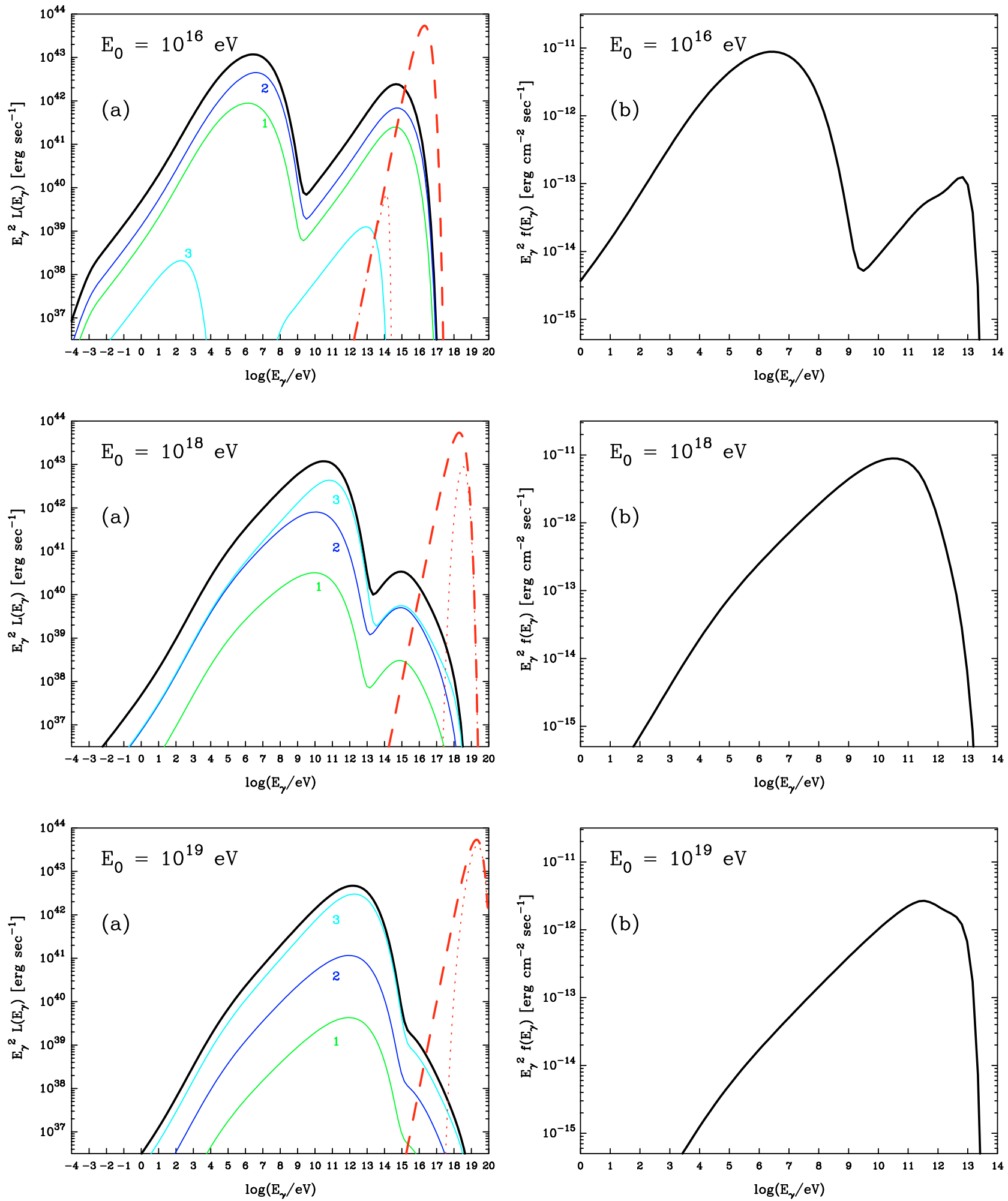

Fig. 5. Left panels a): non-thermal luminosities of a spherically symmetric galaxy cluster of radius $1.5 \mathrm{Mpc}$. The radiation is produced via conversion of VHE $\gamma$-rays into electron-positron pairs and subsequent synchrotron and IC radiation of secondary electrons. It is assumed that $\gamma$-rays with a spectrum given by Eq. (1) with $\Gamma=0, \beta=1$, and (i) $E_{0}=10^{16} \mathrm{eV}$, (ii) $10^{18} \mathrm{eV}$ and (iii) $10^{19} \mathrm{eV}$, have been injected into ICM by a central source during the last $10^{7}$ years. The spectra of the primary $\gamma$-ray source are shown by thick dashed lines. Dotted lines show the spectra of the primary $\gamma$-rays after propagating of $1.5 \mathrm{Mpc}$ (at the edge of the cluster). The area between the dashed and dotted lines indicates the amount of energy absorbed and then reradiated in the cluster volume. The luminosity of the primary VHE $\gamma$-ray source is assumed to be $10^{44} \mathrm{erg} / \mathrm{s}$. The magnetic field in the cluster is assumed to be $B=1 \mu \mathrm{G}$. The luminosities of three different zones of the cluster are shown with thin solid lines: $1\left(R=0-10^{-3} \mathrm{Mpc}\right), \mathbf{2}(R=0.01-0.04 \mathrm{Mpc})$ and $\mathbf{3}(R=0.44-1.5 \mathrm{Mpc})$. The overall luminosity of the cluster is shown by the thick solid line. Right panels b): expected energy fluxes of non-thermal radiation corresponding to the luminosities shown in the right panels, calculated for a cluster at a distance of $100 \mathrm{Mpc}$, after correction for the intergalactic absorption. 


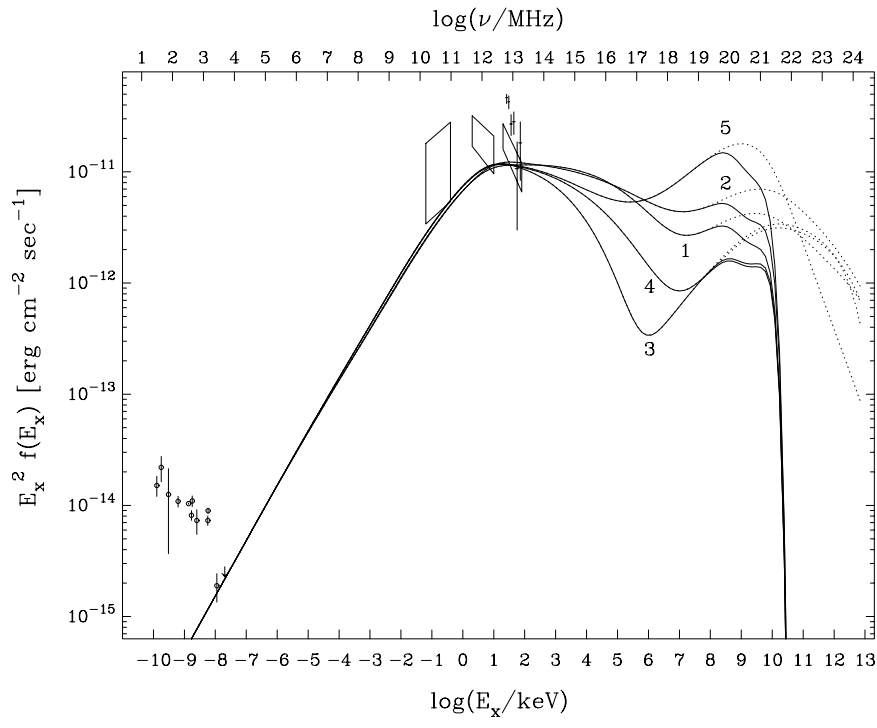

Fig. 6. Energy fluxes of non-thermal radiation from the Coma cluster initiated by products (electrons, positrons, $\gamma$-rays) of decays of super-heavy "X"-particles. The spectra are calculated for different fragmentation functions, intracluster magnetic field, and $m_{\mathrm{X}}$ presented in Table 1. The reported fluxes at radio EUV and X-rays are same as in Fig. 2. The solid lines represent spectra corrected for intergalactic $\gamma$-ray absorption. Unabsorbed spectra are shown by dashed lines.

are presented in Fig. 6. The model parameters are presented in Table 1.

Since the relic X-particles have a cosmological origin, we must assume that the process is continuous over the age of the cluster of about $1 / H_{0} \sim 10^{10} \mathrm{yr}$. During this period the electrons cooled to low energies and thus produced synchrotron radio emission. This provides a strict upper limit to the rate of decay of X-particles to prevent overproduction of radio emission.

The results presented in Fig. 6 show that with a normalization at the radio flux at $\mathrm{GHz}$ frequencies, and for chosen values for $B$ and $m_{\mathrm{X}}$, all five approximations predict EUV and X-ray fluxes which marginally agree with observations. However, the absolute normalizations used in Fig. 6 imply a very high rate of appearance of the highest energy electrons and $\gamma$-rays. The same rate of decay/annihilation of primordial massive particles in the Halo of our Galaxy would result in 3 orders of magnitude higher flux of the highest energy particles than the observed flux of cosmic rays. In order to avoid overproduction of the highest energy cosmic rays, one has to assume a higher density of X particles in the Coma cluster. While in the case of annihilation the density should be $\sim 30$ times higher than in the Galactic Halo, in the case of particle decays the density should be $\sim 1000$ times higher. Either case cannot be easily accommodated within the current dark matter halo formation theories.

\section{Discussion}

The "excess" EUV and hard X-ray emission reported from several galaxy clusters is generally thought to be due to nonthermal processes in the ICM. However, interpretation of this radiation within the most favored inverse Compton model poses serious difficulties. This model not only requires huge
Table 1. Model parameters.

\begin{tabular}{cll}
\hline \hline $\begin{array}{c}\text { Fragmentation } \\
\text { function }\end{array}$ & $B$ & $m_{\mathrm{X}}$ \\
\hline 1 & $1.8 \mu \mathrm{G}$ & $2 \times 10^{12} \mathrm{GeV}$ \\
2 & $3.0 \mu \mathrm{G}$ & $4 \times 10^{12} \mathrm{GeV}$ \\
3 & $2.0 \mu \mathrm{G}$ & $6 \times 10^{11} \mathrm{GeV}$ \\
4 & $1.8 \mu \mathrm{G}$ & $4 \times 10^{12} \mathrm{GeV}$ \\
5 & $1.8 \mu \mathrm{G}$ & $2 \times 10^{13} \mathrm{GeV}$ \\
\hline
\end{tabular}

energy in accelerated particles, but also assumes intracluster magnetic fields significantly below the values derived from RM probes of galaxy clusters.

The synchrotron radiation of multi-TeV electrons which proceeds with almost 100 per cent efficiency seems a more viable channel for production of the "excess" EUV and X-ray emission. The challenge of this mechanism is the required energy of electrons $-100 \mathrm{TeV}$ or more. The lifetime of these electrons and correspondingly their propagation is very short. No acceleration mechanism has been suggested so far that could overcome the severe radiative losses and boost the electrons to such high energies. Moreover, such accelerator(s) should "operate" on $\gg 1 \mathrm{kpc}$ scales in order to fill the ICM with multi-TeV electrons.

In this paper we propose that these electrons have a secondary origin, namely we assume that the EUV and X-ray "excess" emission is the result of synchrotron radiation of secondary ultra-relativistic electrons introduced throughout the cluster continuously (in time and space) via interactions of very high energy $\gamma$-rays with photons from the $2.7 \mathrm{~K} \mathrm{CMBR}$ and diffuse infrared background radiation.

Of course, generation of extremely high energy photons itself is not a trivial process, and this assumption poses the main challenging question for the model. On the other hand, we know that the $\gamma$-ray spectra of BL Lac objects like Mkn 421 and Mkn 501 do extend to multi-TeV energies and do not show the tendency, after correction for the intergalactic absorption, to have a cutoff at least up to $20 \mathrm{TeV}$ (see e.g. Aharonian et al. 2001). Therefore we may speculate that the energy spectra of these objects extend to $100 \mathrm{TeV}$ or beyond. It has been recently argued that $\gamma$-rays of much higher energies can be produced in more powerful AGN (e.g. Neronov et al. 2002; Atoyan \& Dermer 2003), or immediately in the intracluster medium due to interactions of the highest energy cosmic rays with surrounding photon fields (Aharonian 2002). In this case, because the $\gamma$-ray spectra extend well beyond $10^{15} \mathrm{eV}$, the maximum of synchrotron radiation of the secondary electrons may appear in the $\gamma$-ray domain.

Also, extremely high energy $\gamma$-rays could be linked to the decay products of hypothetical super heavy particles from Dark Matter Halos. In this case the decay products $-\gamma$-rays and electrons (positrons) - are produced with energies above $10^{21} \mathrm{eV}$. Their interaction with the surrounding photon and magnetic fields leads to the appearance of the second generation of $\gamma$-rays with energies typically exceeding $100 \mathrm{TeV}$. The latter effectively interact with the diffuse extragalactic photon fields, resulting in second generation electrons and positrons. 
Thus, the synchrotron radiation of these electrons will appear in the X-ray domain.

Above we applied this scenario for the specific case of Coma - a relatively nearby $(d \simeq 100 \mathrm{Mpc}$ ) galaxy cluster showing the strongest evidence of non-thermal emission in the overall EUV and X-ray bands. The numerical calculations demonstrate that the reported EUV and X-ray data can be adequately explained, within experimental uncertainties, for both high and low intracluster magnetic fields (see Figs. 2 and 3). However the value of the magnetic field has a much stronger impact on the $\gamma$-ray fluxes produced through the inverse Compton scattering of the same electrons. To reduce the inverse Compton component of the radiation to $\sim 10^{-11} \mathrm{erg} / \mathrm{cm}^{2} \mathrm{~s}$ (otherwise a positive signal would be seen in the HEGRA data) one must assume that the magnetic field exceeds $3 \mu \mathrm{G}$. This agrees very well with the RM probes of Coma (Kim et al. 1990; Feretti et al. 1995). This also makes more efficient the production of EUV and X-rays because in this case all the kinetic energy of electrons is released through the synchrotron channel.

On the other hand, even in the case a of high intracluster magnetic field one may expect quite large fluxes of direct (primary) $\mathrm{TeV} \gamma$-ray emission. In particular, Eq. (1) with $\Gamma=2$ predicts quite high direct $\mathrm{TeV}$ emission, even after correction for significant intergalactic absorption. This flux can be suppressed assuming that $\gamma$-rays are produced in jets at large angles towards the observer (this would obviously imply that $\gamma$-rays are produced by a single or by a small number of AGN). Another possibility would be a much harder spectrum of primary $\gamma$-rays (or the existence of a low energy cutoff) below $100 \mathrm{TeV}$. While these $\gamma$-rays do not interact effectively with diffuse background photons within $1 \mathrm{Mpc}$ scales (and therefore they do not have significant impact on the spectrum of synchrotron radiation), this assumption can dramatically reduce the flux of primary $\gamma$-rays at $\mathrm{TeV}$ and lower energies.

The production of EUV and X-ray fluxes through synchrotron radiation requires electrons from 10 to $1000 \mathrm{TeV}$. Thus, the spectrum of primary $\gamma$-rays should extend to $1000 \mathrm{TeV}$ or so. Such energetic $\gamma$-rays can be produced in individual galaxies, in particular in AGN, most likely due to proton-proton or proton-photon interactions. In both cases $\gamma$-ray emission is accompanied by high energy neutrinos of comparable flux. In contrast to $\geq 10 \mathrm{TeV} \gamma$-rays, which are absorbed in the intergalactic medium, the neutrinos freely propagate through extragalactic photon fields and reach us. The expected flux of these energetic neutrinos is expected at the same level as for $\gamma$-rays if the latter are not absorbed in the intergalactic medium, i.e. at the level of $10^{-10} \mathrm{erg} / \mathrm{s}$. These fluxes could be detected by planned high energy neutrino detectors (see e.g. Halzen \& Hooper 2002), unless the primary $\gamma$-rays and, therefore, also neutrinos are not produced in the jets at large angles to the observer.

The maximum of synchrotron radiation strongly depends on the energy cutoff in the primary $\gamma$-ray spectrum, $(h v)_{\max } \propto$ $E_{0}^{2}$. If $E_{0}$ significantly exceeds $10^{15} \mathrm{eV}$, the synchrotron peak will be shifted to the $\gamma$-ray domain, from $\mathrm{MeV}$ to $\mathrm{TeV}$ energies, depending on $E_{0}$. If so, this scenario can compete with IC models of $\gamma$-radiation of clusters of galaxies (Keshet et al. 2003; Miniati 2003; Gabici \& Blasi 2003). However, while the $\gamma$-ray spectra in the the IC models hardly could extend beyond $100 \mathrm{GeV}$ (unless particle diffusion in the unshocked region proceeds in the Bohm regime, which however seems quite unlikely), in the proposed scenario of synchrotron radiation of electrons of "photonic" origin, the synchrotron peak could be easily shifted to $\mathrm{TeV}$ energies, assuming that the cutoff energy in the "primary" $\gamma$-ray spectrum exceeds $10^{18} \mathrm{eV}$. The proposed mechanism works with very high efficiency, providing $\mathrm{GeV} / \mathrm{TeV}$ peak luminosities at the level of $10^{43} \mathrm{erg} / \mathrm{s}$ for a relatively modest luminosity of about $10^{44} \mathrm{erg} / \mathrm{s}$ in the primary $\geq 10^{18} \mathrm{eV}$ photons produced in all individual objects belonging to the cluster. If so, one should expect a non-negligible contribution of $\gamma$-rays from rich clusters of galaxies into the diffuse extragalactic $\gamma$-ray background. However, it is difficult to give qualitative estimates of this contribution given uncertainties in the genuine extragalactic $\gamma$-ray background due to contamination caused by diffuse galactic radiation from high latitudes (Strong et al. 2000).

In this paper we investigated the interesting possibility that primary $\gamma$-rays may originate from the decay products of relic heavy particles like topological defects or WIMPs. Although this hypothesis can (marginally) fit the reported EUV and X-ray spectra from Coma, it requires strong enhancement, by a factor of 30 to 1000 , of the dark mater density in Coma compared to the Halo around our Galaxy. This makes the hypothesis of a cosmological origin of primary $\gamma$-rays quite unlikely.

We performed detailed numerical calculation in the framework of the proposed model for the Coma cluster, which shows so far the strongest evidence for non-thermal activity in the intracluster medium. On the other hand, the mechanism discussed in this paper may have a non-negligible impact on radiation properties of other clusters of galaxies, especially those that host strong central AGN or radiogalaxies, like M87 in the Virgo cluster or NGC 1275 in Perseus. If one assumes that these objects emit very energetic $\gamma$-rays, we should expect, in addition to the thermal component of X-rays, also non-thermal synchrotron radiation produced by secondary, pair-produced electrons. If the energy spectrum of $\gamma$-rays extends to $10^{15} \mathrm{eV}$, one may expect a significant amount of X-rays from the core of the cluster, given that the free path of $\sim 10^{15} \mathrm{eV} \gamma$-rays is only $\sim 10 \mathrm{kpc}$. If so, this hypothetical component of radiation should be taken into account in the treatment of X-ray phenomena from clusters of galaxies, in particular in the context of the ongoing debates concerning the "cooling flows" (e.g Fabian et al. 2002; Colafrancesco et al. 2003). If identified, this radiation would also help to "recover" information about the $\geq 100 \mathrm{TeV}$ $\gamma$-rays which otherwise would not be visible due to the intergalactic absorption.

Finally we note that the search for synchrotron emission of electrons of "photonic" origin requires its identification and thorough separation from the thermal (optically thin bremsstrahlung) component, taking into account that these radiation components may have rather similar spectral shapes. Both components can be described by a power-law spectrum with "quasi-exponential cutoff"

$F_{v} \sim v^{-\alpha} \exp \left[\left(-v / v_{0}\right)^{\kappa}\right]$. 
However, while the spectral index of thermal bremsstrahlung at energies $h v \ll k T$ is $\alpha_{\text {therm }}=0$, the spectral index of the synchrotron component is close to $\alpha_{\mathrm{syn}} \approx 0.5$. Thus, for the fixed cutoff energy $h v_{0}$, detection of an excess emission below $h v_{0}$ may be an indicator of the synchrotron component.

The cutoff energy for the thermal component is directly related to the temperature of the intracluster gas, $h v_{0, \text { th }} \simeq k T$ (typically a few $\mathrm{keV}$ ), and the spectrum in the cutoff region drops exponentially $(k=1)$. The synchrotron cutoff energy depends on the intracluster magnetic field $B$ and on the cutoff energy in the primary photon spectrum $E_{0}: h v_{0, \text { synch }} \simeq$ $10(B / 3 \mu \mathrm{G})\left(\mathrm{E}_{0} / 10^{15} \mathrm{TeV}\right)^{2} \mathrm{keV}$. For a certain combination of ICMF and the cutoff energy in the spectrum of primary $\gamma$-rays, the corresponding cutoff energy in the spectrum of synchrotron radiation may appear around a few $\mathrm{keV}$, i.e. quite close the the cutoff energy expected in the thermal radiation component. On the other hand the spectrum of the synchrotron radiation in the cutoff region is described by the parameter $\kappa \approx \beta / 2$, therefore it drops as a simple exponent only for a specific distribution of primary $\gamma$-rays given by Eq. (1) with $\beta=2$.

Thus, although the position of the cutoff energy in the synchrotron spectrum may appear in the typical range for thermal emission of several keV, the spectrum both below and above the cutoff energy generally should deviate from the thermal spectrum. It would be a challenge to search for such a component of radiation from the cores of galaxy clusters.

Acknowledgements. The authors thank V. Petrosian for interesting discussion. AT acknowledges the financial support from INTAS, grant YSF-2002-229, and Russian Federation President Grant Program, grants MK-895.2003.02, NSh-388.2003.2.

\section{References}

Aharonian, F. A. 2002, MNRAS, 332, 215

Aharonian, F. A., Akhperjanian, A. G., Barrio, J. A., et al. 2001, A\&A, 366,62

Aharonian, F. A., Timokhin, A. N., \& Plyasheshnikov, A. V. 2002, A\&A, 384, 834

Atoyan, A. M., \& Dermer, C. D. 2003, ApJ, 586, 79

Atoyan, A. M., \& Völk, H. J. 2000, ApJ, 535, 45

Berezinsky, V., \& Kacherliess, M. 2001, Phys. Rev. D, 63, 034007

Berezinsky, V. S., Blasi, P., \& Ptuskin, V. S. 1997, ApJ, 487, 529

Berghöfer, T. W., \& Bowyer, S. 2002, ApJ, 565, L17

Berghöfer, T. W., Bowyer, S., \& Korpela, E. 2000, ApJ, 535, 615

Bhattacharjee, P., \& Sigl, G. 2000, Phys. Rep., 327, 109

Birkel, M., \& Sarkar, S. 1998, APh, 9, 297

Blasi, P. 2000, ApJ, 532, L9
Blasi, P. 2002, APh, 16, 429

Bowyer, S., \& Berghöfer, T. W. 1998, ApJ, 506, 502

Bowyer, S., Berghöfer, T. W., \& Korpela, E. J. 1999, ApJ, 526, 592

Bowyer, S., Lampton, M., \& Lieu, R. 1996, Science, 274, 1338

Brunetti, G., Setti, G., Feretti, L., \& Giovannini, G. 2001, NewA, 6, 1

Clarke, T. E., Kronberg, P. P., \& Böhringer, H. 2001, ApJ, 547, L111

Colafrancesco, S., Dar, A., \& De Rujula, A. 2003 [astro-ph/0304444]

Deiss, B. M., Reich, W., Lesch, H., \& Wielebinski, R. 1997, A\&A, 321, 55

Dogiel, V. A. 2000, A\&A, 357, 66

Enßlin, T. A., Lieu, R., \& Biermann, P. L. 1999, A\&A, 344, 409

Enßlin, T. A., \& Sunyaev, R. A. 2002, A\&A, 383, 423

Fabian, A. C., Allen, S. W., Crawford, C. S., et al. 2002, MNRAS, 332, L50

Feretti, L., Dallacasa, D., Giovannini, G., \& Tagliani, A. 1995, A\&A, 302,680

Fusco-Femiano, R., Dal Fiume, D., De Grandi, S., et al. 2000, ApJ, 534, L7

Fusco-Femiano, R., Dal Fuime, D., Feretti, L., et al. 1999, ApJ, 513, L21

Gabici, S., \& Blasi, P. 2003 [astro-ph/0306369]

Giovannini, G., Tordi, M., \& Feretti, L. 1999, NewA, 4, 141

Halzen, F., \& Hooper, D. 2002, RPPh, 65, 1025

Hill, C. T. 1983, Nucl. Phys. B, 224, 469

Hwang, C. 1997, Science, 278, 1917

Inoue, S., \& Sasaki, S. 2001, ApJ, 562, 618

Kaastra, J. S., Lieu, R., Mittaz, J. P. D., et al. 1999, ApJ, 519, L119

Keshet, U., Waxman, E., Loeb, A., Springel, V., \& Hernquist, L. 2003, ApJ, 585, 128

Kim, K.-T., Kronberg, P. P., Dewdney, P. E., \& Landecker, T. L. 1990, ApJ, 355, 29

Liang, H., Dogiel, V. A., \& Birkinshaw, M. 2002, MNRAS, 337, 567

Lieu, R., Ip, W.-H., Axford, W. I., \& Bonamente, M. 1999, ApJ, 510, L25

Lieu, R., Mittaz, J. P. D., Bowyer, S., et al. 1996, Science, 274, 1335

Loeb, A., \& Waxman, E. 2000, Nature, 405, 156

Miniati, F. 2002, MNRAS, 337, 199

Miniati, F. 2003, MNRAS, 342, 1009

Neronov, A., Semikoz, D., Aharonian, F., \& Kalashev, O. 2002, PhRvL, 89, 51101

Petrosian, V. 2001, ApJ, 557, 560

Rephaeli, Y. 1977, ApJ, 212, 608

Rephaeli, Y., \& Gruber, D. 2002, ApJ, 579, 587

Rephaeli, Y., \& Gruber, D. E. 1988, ApJ, 333, 133

Sarazin, C. L., \& Kempner, J. C. 2000, ApJ, 533, 73

Sarazin, C. L., \& Lieu, R. 1998, ApJ, 494, L177

Strong, A. W., Moskalenko, I. V., \& Reimer, O. 2000, ApJ, 537, 763

Tsay, M. Y., Hwang, C., \& Bowyer, S. 2002, ApJ, 566, 794

Volk, H. J., Aharonian, F. A., \& Breitschwerdt, D. 1996, Space Sci. Rev., 75, 279 